\title{
Managing Urban Complexity through Decision Coordination
}

\author{
Shih-Kung Lai \\ College of Architecture and Urban Planning, Tongji University, Shanghai, China \\ Email: lai@tongji.edu.cn
}

How to cite this paper: Lai, S.-K. (2020). Managing Urban Complexity through Decision Coordination. Modern Economy, 11, 239-248.

https://doi.org/10.4236/me.2020.112021

Received: December 28, 2019

Accepted: February 2, 2020

Published: February 5, 2020

Copyright () 2020 by author(s) and Scientific Research Publishing Inc. This work is licensed under the Creative Commons Attribution International License (CC BY 4.0).

http://creativecommons.org/licenses/by/4.0/

\section{(c) (i) Open Access}

\begin{abstract}
The purpose of the paper is to identify conceptually effective action modes for coordinating decisions, given a scope of interdependent decisions. More specifically, the paper addresses a fundamental question about coordinating decisions: given a finite set of decisions, how should we coordinate these decisions through plans, administration, regulations, and governance? To address the question, we analyze axiomatically structures of decisions through binary relations. There are four types of such relations, namely, dependence, independence or converse of dependence, mutual dependence, and mutual independence. Each relation constitutes an ordering with associated properties. Structural characteristics of the four types of relations can be investigated. A scope of coordinating decisions is defined as the transitive closure of a binary relation and a coordination mode is defined as the choice function within the scope through which the coordination mode results in the best outcome. Possible extensions of the conceptual framework are discussed.
\end{abstract}

\section{Keywords}

Plans, Administration, Regulations, Governance, Urban Complexity

\section{Introduction}

Cities are the outcomes of interacting decisions made by many partially independent agents, including individuals, families, firms, developers, organizations, local governments, etc. In order to improve urban development, these decisions must be coordinated to some extent through different mechanisms. The objective of the present paper is to identify conceptually effective decision coordination modes for urban complexity, given a scope of interdependent decisions. More specifically, the paper addresses a fundamental question about coordinating decisions: given a finite set of interdependent decisions, how should we 
identify appropriate scopes and, at the same time, look for useful, not necessarily optimal, modes of decision coordination? Decision coordination is not a new idea, but presented in the context of urban complexity, this paper might shed some new light on how cities should be managed effectively.

It is recognized that there is order embedded in urban complexity and such order is spontaneous (Lai and Han, 2014). However, the spontaneous order may not be efficient. Consider natural vs. man-made cities. The geometric order that emerges in the former through self-organization is mainly fractal, while the geometric order imposed in the latter through planning is largely Euclidean. A city cannot escape from the natural order, but the man-made order makes it an efficient place to live. Therefore, the greatest challenge for managing urban complexity is to balance the two types of order.

Traditionally, cities are managed through planning which brings about the man-made order. Interpretations about planning have proliferated in the literature based on various perspectives, including economics (e.g., Intriligator \& Sheshinski, 1986), game theory (e.g., Knaap et al., 1998), sociology (Friedmann, 1978), mathematical programming (Hopkins, 1974), and ecology (Steiner, 1991). Most of these works focus, however, on the meaning of planning per se at the philosophical level (e.g., Faludi, 1973), on the implications of planning in the context of social structure at the abstract level (e.g., Friedmann, 1978), on solving planning problems at the methodological level (e.g., Friend \& Hickling, 2005), and on the conducting of planning in a particular domain at the empirical level (e.g., Chapin \& Kaiser, 1979). They tend to abstract planning away from the empirical world and treat it as an ideal, artificial, but not necessarily rational, process for attaining some presumed goals. Viewing planning as a natural way for people of dealing with problems encountered, we argue that planning, like decision making, is a behavior common to all human beings, not just a contrived idea. The study of planning behavior is, therefore, central to planning research.

However, planning affects the urban development process through information (Hopkins, 2001) and can thus only accomplish certain things. With the advance of technology, cities today are more complex and compressed in time and space that defy the traditional planning techniques to deal with. Though much has been said about the complexity of cities, relatively little has been argued for how we should deal with that complexity (e.g., West, 2017). Therefore, managing urban complexity requires more than planning. Other modes of managing urban complexity include administration, regulations, and governance. All the four modes of intervention are considered in this paper as coordinating decisions. Improving human settlements requires all four modes of decision coordination to work together (Hopkins, 2001).

In Section 2, we clarify four types of decision coordination modes for urban complexity. In Section 3, we define carefully commonly used terms in decision coordination. In Section 4, we delve into structural characteristics of sequential decisions. In Section 5, we depict a conceptual framework for four different types of decision coordination in light of a specific model of urban complexity. 
In Section 6, we argue for possible extensions of the conceptual framework. In Section 7, we conclude.

\section{Actions, Decisions, Consequences, and Coordination}

In order to make the conception depicted in Section 2 more concrete and operational, consider a decision maker facing a complex set of interdependent decisions each of which is composed of a subset of actions to choose from. How would the decision maker coordinate these decisions to cope with the complexity to make progress? A natural response is to prioritize these decisions independently before making the first move. When these decisions are interdependent through consequences of actions and through budgetary limitations, prioritization among decisions may not yield the best move. It would be more effective to coordinate these decisions that are interrelated before taking the first action. But there is no analytic backing for this coordination, or it is only at best slighted in seemingly logical frameworks (e.g., Friend \& Hickling's (2005) Strategic Choice Approach).

Most decision theories consider the situations where decisions are given, regardless of whether these decisions are interrelated (e.g., Keeney and Raiffa, 1976). Considering interlinked decisions in sequence is only slighted in most decision theory texts (e.g., Hammond, Keeney, \& Raiffa, 1999). There is a need for guiding decision makers normatively as to how sets of interlinked decisions should be considered, that is coordination of decisions should be made, in light of the interdependence of decisions. The paper addresses this question directly and conceptually based on mathematics of decision theory. We set aside at present how the dynamics of decisions evolves, that is how decisions are made and interrelated in time. Here we use the terms interlinked, interrelated, and interdependent interchangeably.

Before we proceed, some daily used terms need to be carefully defined. An action is a move to act, a commitment of a decision. Actions may or may not be interrelated. Interrelatedness of actions is defined by whether the activation of one action affects the values of the consequences of another action. A decision is composed of a set of actions to choose from. Decisions themselves may or may not be interdependent depending on whether the actions in the two decisions are interrelated. A consequence is the result of an action in combination with the effect of the complex system, a collective result from enacting interrelated decisions.

\section{Planning, Administering, Regulating, and Governing Cities}

Coordinating decisions connotes arranging decisions in space and time so that the outcome of such arrangement yields an acceptable level of satisfaction. The decisions are interdependent and can be made by the same decision maker or by other decision makers. There can be four ways of coordinating decisions: planning, administering, regulating, and governing. A plan is a path in a decision 
tree that takes into account possible alternatives and uncertain outcomes and can be analyzed through the decision analysis framework (Hopkins, 2001). Effective administration depends on useful organizational designs in that organizations are manifestations of decision coordination. Plans and organizations thus complement each other. Regulations identify permissible rights for the decision maker to act. Governance implies collective choices and actions. Managing urban complexity requires all four modes of decision coordination; that is planning, administering, regulating, and governing, or PARGing, cities. In particular, plans coordinate decisions through information; administration coordinates decisions through authorities in organizations; regulations coordinate decisions through rights; and governance coordinates decisions through collective choices, all bringing about order in urban complexity. In terms of purposes, plans tend to cope with the problems of dynamics failure; administration deals with the problems of government failure; regulations cope with the problems of market failure in relation to externalities; and governance deals with the problems of market failure in relation to collective goods.

\section{Independent, Dependent, and Interdependent Decisions}

Relations among decisions can best be described by binary relations. There are four types of such relations: independence, dependence, mutual independence, and mutual dependence, among which the dependence relation is the fundamental one from which other relations can be derived. Let $X$ be a finite set of decisions; $A$ a finite set of actions; $C$ a finite set of consequences, and $f$ a function mapping from $X$ to $C$ through $A$.

Definition: Decision dependence

Suppose $x$ and $y \in X$ and are nonempty sets of actions. $x R y$ if and only if the choice of actions in $x$ depends on the consequences of actions in $y$.

It can be concluded that given $x, y \in X$, there are four relational possibilities:

1) $x R y$ and not $y R x$ (dependence, $R$ );

2) not $x R y$ and $y R x$ (converse of dependence, $C$ );

3) $x R y$ and $y R x$ (mutual dependence or interdependence, $T$ );

4) not $x R y$ and not $y R x$ (mutual independence, $I$ ).

In order to prescribe analytically how the decision maker should choose actions from the four relational structures, we need first to explore the characteristics associated with these structures. Following Fishburn (1972), there are five categories of relational properties given a binary relation $R$ on $X$.

1) reflexivity. $x R x$.

2) irreflexivity. not $x R x$.

3) symmetry. $x R y$ implies $y R x$.

4) asymmetry. $x R y$ implies not $y R x$.

5) antisymmetry. $x R y \& y R x$ imply $x=y$.

6) transitivity. $x R y \& y R z$ imply $x R z$.

7) negative transitivity. not $x R y \&$ not $y R z$ imply not $x R z$. Equivalently: $x R z$ implies $x$ Ry or $y R z$. 
8) connectedness (completeness). $x R y$ or $y R x$.

9) weak connecteness. $x \neq y$ implies $x R y$ or $y R x$.

10) $x R y \& z R w$ imply $x R w$ or $z R y$.

11) $x R y \&$ \& $y z$ imply $x R w$ or $w R z$.

It can be easily shown through games that the dependence relation is reflexive. That is, the choice of a decision depends on that of itself or $x R x$. The dependence relation is also transitive in that if $x R y$ and $y R z$ then $x R z$. Therefore, $R$ is a partial preorder. Denote $R$ by $\succ$ and we have the following properties of the preorder (Debreu, 1987). Let $X, y \in X$ and $\geq$ is a partial preorder on $X$.

1) By definition, $y \geq x$ means $x \leq y$.

2) $x \leq y$ and $y \leq x$ is written $x \sim y$, meaning that $x$ and $y$ are mutually dependent or interdependent (mutual dependence).

3) $x \leq y$ and not $y \leq x$ is written $x<y$ (or $y>x$ ), meaning that $x$ is strictly dependent or one way dependent on y (converse of dependence).

4) Relations of pairs other than the ones mentioned above are mutually independent (mutual independence).

It can be easily shown through games that the independence relation is irreflexive. That is, the fact that the choice of a decision depends on the consequences of itself or $x R x$ implies that not $x C x$. It is also transitive in that if $x C y$ and $y C z$ then $x C z$. Therefore, $C$ is a strict partial order. Since $C$ is a strict partial order, according to Zorn's lemma, it would be interesting to examine the properties of the extension structure. By similar arguments, the mutual dependence relation and the mutual independence relation are partial preorder and strict partial order respectively.

\section{A Conceptual Framework for Identifying Scopes and Coordinating Decisions}

A scope of coordinating decisions is a transitive closure of $R$ on $X$ where $\mathrm{U}=R$ $\cup R R \cup R R R \cup R R R R \ldots$ is a strict partial order because by definition not $x R x$ implies not $y R x$ and therefore not $x R y$. Any decision outside the scope is not worth considering in the coordinating mode because it can be evaluated independent of other decisions. In other words, we are only interested in dependent decisions. The scope for decision coordination can be identified by contiguity analyses. For example, the first order of a contiguity matrix is composed of $0 \mathrm{~s}$ and $1 \mathrm{~s}$ in that a " 1 " in the cell represents the decision in the row is dependent of the decision in the column. The second order of the contiguity matrix results from the multiplication of the matrix by itself. The $1 \mathrm{~s}$ in the resulting matrix indicate that the decisions in the corresponding rows are dependent of those in the corresponding columns, but indirectly by two steps of transitivity. The way of constructing contiguity matrices can go on to the infinity and the transitive closure is the union of all pairs of dependent decisions over all the steps of transitivity.

Any scope implies a set of possible modes of decision coordination each of which is composed of a set of dependent decisions. A choice function $\Phi$ on a 
scope $\mathrm{U}$ is to select the coordinating mode that yields the best outcome within that scope. Thus the choice of the scope and that of a coordinating mode confounds each other. We cannot expect to make an optimal coordinating mode in a complex system, but we can at least set up appropriate search strategies considering the scope and associated possible coordinating modes at the same time. Lai (2002) proved the optimal search strategy for planning in terms of information gathering in a one-person organization. In particular, information structures that are degarbling and accurate should be sought by the planner in order to yield the best outcome. We focus here however on interdependence among decisions.

There are many ways of modeling cities. The model on which the ensuing argument is based is called the spatial garbage can model (Lai, 2006). In essence, the model looks at cities as giant organized anarchies with numerous decisions, including mainly activities and constructions. A city in the model is conceived of as five seemingly independent streams of entities: decision makers, problems, solutions, choice opportunities, and locations. The elements of these entities meet in a random fashion over time and if decision makers, problems, and solutions collide and are thrown into choice opportunities located in a particular place to fulfill some structural constraints, then something happens. We do not claim that the spatial garbage can model is the model for urban development, but that the model has been validated partially through empirical studies (Lai et al., 2018).

\subsection{Coordinating Decisions through Plans}

In the context of the spatial garbage can model, planning is theoretically equivalent to arranging choice opportunities over time in order to yield the optimal amount of net energy. Energy stands for resources required to solve problems, such as time, labor, and money. A planning tool for making multiple, linked decisions has been developed based on this definition of planning (Han \& Lai, 2011 and Lai \& Huang, 2017). Conceptually, a plan is an assignment of decision makers, problems, and solutions to choice opportunities forming a network of these elements which can then be solved by a linear programming model (Lai and Huang, 2017). A plan thus defined is a set of interdependent decisions. In addition, plans coordinate decisions through information by revealing the contents and thus intents of these plans to other decision makers in order to affect their behavior. A plan is a sequence of decisions as to what to do now in light of what to do in the future. If a decision in a plan is interdependent of another decision in another plan, the planner who owns the latter plan is motivated to revise his/her plans in light of the information acquired about the former plan. The revised plans will affect directly or indirectly the ensuing chain of interdependent decisions in the transitive closure. Such interaction of plans could be formalized by utility theory (Lai, 2018) and the logic of making and using plans is rigorously depicted by Hopkins (2001). 


\subsection{Coordinating Decisions through Administration}

Administration coordinates decisions through authorities in organizations by structuring these decisions in a particular pattern, such as a tree, hierarchy, or network, so that they can function collectively to achieve a common goal. Organizations are recognized as organized anarchies in that the processes of decision making are partially chaotic. Structures of planning can be imposed on the seemingly chaotic processes in order to change how things work (Lai, 1998, 2003). In particular, cities can be conceived of as giant organized anarchies and by adding an element of space into the garbage can model (Cohen et al., 1972), Lai (2006) argued that institutional design for urban development is no less important than spatial design. As a result, plans and organizations complement each other with a distinction that the former change behavior through information, while the latter through authority. A change in the decision structures, or organizations, of cities will result in the change in the outcome of urban development through affecting the ensuing chain of interdependent decisions in the transitive closure.

\subsection{Coordinating Decisions through Regulations}

Regulations coordinate decisions through rights delineation by identifying what can or cannot be done in a particular situation so that decisions made by many persons become predictable and manageable. Rights cannot be delineated completely so even with regulations set up, there will always be property rights dissipated in the public domain for agents to acquire. In the context of the spatial garbage can model, a change in the structure constraints, or institutions, of cities will result in the change in the outcome of urban development through affecting the ensuing chain of interdependent decisions in the transitive closure.

\subsection{Coordinating Decisions through Governance}

Governance coordinates decisions through social or collective choices by designing mechanisms that aggregate preferences in order to make collective decisions for groups. According to Arrow (1951), there exists no such a mechanism that satisfies the fundamental conditions of democracy. Collective decisions are made for groups that are associated with plans. In the context of the spatial garbage can model, a group or coalition is a set of decision makers associated with a set of interdependent choice opportunities that function collectively as a plan. This group or coalition of decision makers may make collective decisions regarding collective goods provision, such as plans. This collective decision may affect the interdependent decisions that are owned by the decision makers and thus result in the change in the ensuing chain of interdependent decisions in the transitive closure.

Three structural distributions of dependent decisions can be distinguished: independent decisions, uniform distribution of dependent decisions, and clustered distribution of dependent decisions. In the distribution of independent de- 
cisions, all decisions in the finite set are independent of each other. There is no gain for coordinating decisions in this case. In the uniform distribution of dependent decisions, all the decisions are dependent of each other, and coordinating decisions implies that the coordinating mode must be complete in that all decisions must be considered in decision coordination in order to gain. In the clustered regime, decisions are partially dependent of each other, and the scope of coordinating decisions is of paramount importance. The question of how coordinating modes should be made in order to gain under the clustered distribution can be addressed directly in the proposed analytic framework. Evidence shows that the distribution of the interdependence of decisions is likely to be hierarchical or complex in space and time (e.g., Wolfram, 1994; Simon, 1998), which implies that coordinating decisions should be a continuing activity.

\section{Possible Extensions to Urban Management}

Three possible extensions of the analytic framework can be made in relation to urban management. Firstly, urban development processes can be characterized by at least four aspects: Interdependence, Irreversibility, Indivisibility, and Imperfect Foresight or 4 I's (Hopkins, 2001). The focus in the proposed analytic framework is on interdependent decisions and we have described to some extent what interdependence means. Irreversibility means decisions cannot be reversed without incurring some cost. Indivisibility means that decisions cannot be made in arbitrarily small scales. Imperfect foresight means that we cannot anticipate consequences of actions in decisions perfectly. The proposed analytic framework can incorporate the other three aspects incrementally in order to identify conditions where coordinating decisions can gain (c. f., Lai, 2018).

Secondly, automata theory is an extension of the current framework to consider the dynamics of interdependent decisions. For example, viewing decisions, problems, choice opportunities, and solutions as separate, interacting elements in an organization, Cohen et al. (1972) ran a simulation of garbage can model to investigate how organizational choice behavior was sensitive to externally controlled variables. Extensions of that simulation were made by Lai $(1998,2001)$ to explore into effects of making plans on the garbage can decision processes. Formalizing axiomatically the garbage can processes is possible based on automata theory. In particular, decisions, problems, choice opportunities, and solutions can be considered as alphabets of a language of a finites automata machine the states of which are composed of whether to enact a decision. Using automata theory, we could construct guidelines of controlling discrete events, or planning, in order to impose some structures on the seemingly chaotic processes (Lin, 1991).

Finally, we have argued that decisions are likely to be related in hierarchical, complex structures. The implication is that decisions could be related to each other in a distribution where there are many clustered, mutually interdependent decisions. Coordinating decisions is most likely to gain in such distribution. This argument is reminiscent of the recent development in systems theory where the 
emergence of self-organization of patterns resulting from interacting agents is the rule, rather than exception (e.g. Holland, 1998). In order to render our analytic framework useful, there is a need for providing evidence that the hierarchical, complex structure of dependent decisions is a condition that exists in all complex systems.

\section{Conclusion}

When faced with many, clustered, and mutually dependent decisions, decision makers lack useful analytic skills in coping with such complexity. Based on the analytic framework of mathematics of decision theory, the paper focuses on how to make sense of such complexity by identifying the scopes where useful coordinating modes are likely to be made. The insights into how to act in a web of interrelated decisions thus gained can back the notion that decision coordination matters. Axiomatic extensions of the analytic framework proposed to aspects in urban management other than interdependence can be made from this starting point. Much work remains to be done.

\section{Conflicts of Interest}

The author declares no conflicts of interest regarding the publication of this paper.

\section{References}

Arrow, K. J. (1951). Social Choice and Individual Values. New York: John Wiley \& Sons.

Chapin, F. S., \& Kaiser, E. J. (1979). Urban Land Use Planning (3rd ed.). Urbana, IL: University of Illinois Press.

Cohen, M. D., March, J. G., \& Olsen, J. P. (1972). A Garbage Can Model of Organizational Choice. Administrative Science Quarterly, 17, 1-25. https://doi.org/10.2307/2392088

Debreu, G. (1987). Theory of Value: An Axiomatic Analysis of Economic Equilibrium. London: Yale University Press.

Faludi, A. (1973). Planning Theory. Oxford: Pergamon.

Fishburn, P. C. (1972). Mathematics of Decision Theory. Paris: Mouton.

Friedmann, J. (1978). Innovation, Flexible Response and Social Learning: A Problem in the Theory of Meta-Learning. In R. W. Burchellm, \& G. Sternlieb (Eds.), Planning Theory in the 1980s. New Brunsky, NJ: The Center for Urban Policy Research.

Friend, J., \& Hickling, A. (2005). Planning under Pressure: The Strategic Choice Approach. Amsterdam, the Netherlands: Elsevier.

Hammond, J. S., Keeney, R. L., \& Raiffa, H. (1999). Smart Choices: A Practical Guide to Making Better Decisions. Boston, MA: Harvard Business School Press.

Han, H., \& Lai, S. K. (2011). Decision Network: A Planning Tool for Making Multiple, Linked Decisions. Environment and Planning B: Planning and Decision, 38, 115-128. https://doi.org/10.1068/b35153

Holland, J. H. (1998). Emergence: From Chaos to Order. Reading, MA: Helix Books.

Hopkins, L. D. (1974). Plan, Projection, Policy-Mathematical Programming and Planning Theory. Environment and Planning A, 6, 419-430.

https://doi.org/10.1068/a060419 
Hopkins, L. D. (2001). Urban Development: The Logic of Making Plans. New York: Island Press.

Intriligator, M., \& Sheshinsku, E. (1986). Toward a Theory of Planning. In W. Heller, \& R. S. Starrett, (Eds.), Social Choice and Public Decision Making. Cambridge, UK: Cambridge University Press.

Keeney, R. L., \& Raiffa, H. (1976). Decisions with Multiple Objectives: Preferences and Value Tradeoffs. New York: John Wiley \& Sons.

Knaap, G. J., Hopkins, L. D., \& Donaghy, K. P. (1998). Do Plans Matter: A Game-Theoretic Model for Examining the Logic and Effects of Land Use Planning. Journal of Planning Education and Research, 18, 25-34. https://doi.org/10.1177/0739456X9801800103

Lai, S. K. (1998). From Organized Anarchy to Controlled Structure: Effects of Planning on the Garbage-Can Decision Processes. Environment and Planning B, 25, 85-102. https://doi.org/10.1068/b250085

Lai, S. K. (2001). Dose Making Plans Matter in Complex Organizational Systems: An Empirical Examination. Taipei: Paper presented at the Annual Meeting of Regional Science Association.

Lai, S. K. (2002). Information Structures Exploration as Planning for a Single Person Organization. Planning and Markets, 5, 32-41. https://www.researchgate.net/publication/288957967_Information_structures_explorat ion_as_planning_for_a_unitary_Organization

Lai, S. K. (2003). Effects of Planning on the Garbage Can Decision Processes: A Reformulation and Extension. Environment and Planning B: Planning and Design, 30, 379-389. https://doi.org/10.1068/b12981

Lai, S. K. (2006). A Spatial Garbage Can Model. Environment and Planning B: Planning and Design, 31, 141-156. https://doi.org/10.1068/b31111

Lai, S. K. (2018). Why Plans Matter in Cities. Cities, 73, 91-95. https://doi.org/10.1016/j.cities.2017.10.014

Lai, S. K., \& Han, H. (2014). Urban Complexity and Planning: Theories and Computer Simulations. London: Ashgate.

Lai, S. K., \& Huang, J. Y. (2017). Theoretical Foundation of a Decision Network for Urban Development. Frontiers of Information Technology \& Electric Engineering, 18, 1033-1039. https://doi.org/10.1631/FITEE.1510000

Lai, S. K., Kuo, S. C., \& Yu, K. W. (2018). Empirical Validation of the Spatial Garbage Can Model: A Case of Taipei. City and Planning, 45, 1-24. (In Chinese)

Lin, F. (1991). A New Approach to Decision Making: Theory. Unpublished Manuscript.

Simon, H. A. (1998). The Sciences of the Artificial. Cambridge, MA: The MIT Press.

Steiner, F. (1991). The Living Landscape: An Ecological Approach to Landscape Planning. New York: McGraw-Hill.

West, G. (2017). Scale: The Universal Laws of Growth, Innovation, Sustainability, and the Pace of Life in Organisms, Cities, Economies, and Companies. New York: Penguin Press.

Wolfram, S. (1994). Cellular Automata and Complexity: Collected Papers by Stephen Wolfram. Boulder, CO: Westview Press. 